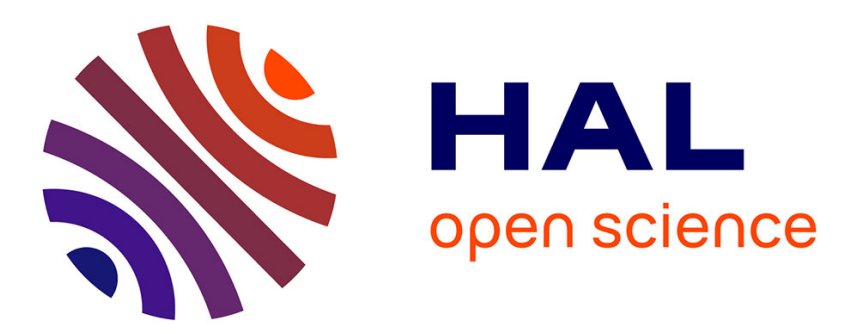

\title{
Dynamic Difficulty Adaptation in Serious Games for Motor Rehabilitation
}

\author{
Nadia Hocine, Abdelkader Gouaich, Stefano A. Cerri
}

\section{To cite this version:}

Nadia Hocine, Abdelkader Gouaich, Stefano A. Cerri. Dynamic Difficulty Adaptation in Serious Games for Motor Rehabilitation. GameDays, Apr 2014, Darmstadt, Germany. pp.115-128, 10.1007/978-3-319-05972-3_13. lirmm-01121647

\section{HAL Id: lirmm-01121647 https://hal-lirmm.ccsd.cnrs.fr/lirmm-01121647}

Submitted on 2 Mar 2015

HAL is a multi-disciplinary open access archive for the deposit and dissemination of scientific research documents, whether they are published or not. The documents may come from teaching and research institutions in France or abroad, or from public or private research centers.
L'archive ouverte pluridisciplinaire HAL, est destinée au dépôt et à la diffusion de documents scientifiques de niveau recherche, publiés ou non, émanant des établissements d'enseignement et de recherche français ou étrangers, des laboratoires publics ou privés. 


\title{
Dynamic Difficulty Adaptation in Serious Games for Motor Rehabilitation
}

\author{
Nadia Hocine, Abdelkader Gouaïch, and Stefano A. Cerri \\ University of Montpellier 2, France \\ \{nadia.hocine, gouaich, cerri\}@lirmm.fr
}

\begin{abstract}
In the last few years, a growing interest has been devoted to improve rehabilitation strategies by including serious games in the therapy process. Adaptive serious games seek to provide the patients with an individualized rehabilitation environment that meets their training needs. In this paper, a dynamic difficulty adaptation (DDA) technique is suggested. This technique focuses on the online adaptation of the game difficulty by taking into account patients' abilities and motivation. The results of the experiment show that the adaptation technique increases the number of tasks, number of successful tasks as well as the movement amplitude during a game session. The technique positively effects the training outcomes of stroke patients, which can help them to recover their functions.
\end{abstract}

Keywords: Adaptation, serious games, stroke

\section{Introduction}

A stroke is among the major causes of adults' disability and death worldwide. According to the location and the size of the brain lesion, the patients may have different combinations of deficits. This includes for example the inability to move one or more limbs, attention deficits, hemineglect and to understand or formulate speech.

Rehabilitation strategies aim to help patients to regain their functions and to independently perform their daily activities. In particular, upper-limb rehabilitation focuses on the enhancement for example of the range of motion, coordination, motion control and muscle strength. They are based on task-oriented strategies in which the patient is asked to perform reaching and grasping activities. With these repetitive activities, patients often become frustrated and tired and their motivation should be supported by the therapist. Indeed, many studies in motor learning showed that the outcomes of the rehabilitation depend on the quality and the amount of physical activities [1] as well as the patients' active participation and engagement during the therapeutic session [2] [3].

Serious games for rehabilitation (or rehabilitation games) can therefore play an important role in providing the patients with a motivating rehabilitation environment in which tasks are adapted to their abilities and training needs. 
They also help therapists to support patients' motivation and to simultaneously supervise different patients.

Many researchers have been interested in identifying what can make the game fun and engaging. In this context, we can find different studies that determine the motivational factors e.g. challenge, curiosity and fantasy [5] as well as the notion of optimal experience as first defined by Csikszentmihalyi [6]. The latter is based on the theory of flow which indicates the full immersion in or engagement with an experience. This state may be reached through a successful tradeoff between the players' skills and challenges.

In this paper, we suggest a difficulty adaptation technique for rehabilitation games. The objective is that the patients do not find the game too easy and thus they may feel bored or too difficult and therefore be frustrated. Maintaining patients' motivation may consequently increase their training outcomes during a therapeutic session.

In the following sections, we first introduce related work on difficulty adaptation in serious games for upper limb rehabilitation. In Section 3, we describe the player's profile used to adapt the game. Then, Section 4 deals with the difficulty adaptation technique. After that, we describe the experimental protocol in Section 5. In Section 6, the results of the experiment are discussed. Finally, we conclude this paper by discussing our findings and initiating our future work.

\section{Related Work}

As patients have limited abilities due to stroke, the difficulty of the game has been considered as an important element to adapt [4] [7]. Difficulty adaptation consists in not only assisting the patient when the task is very difficult, but also challenging him/her in order to increase the training outcomes.

The online difficulty adaptation approach has got the interest of many researchers in rehabilitation games [8] [9] [10] [11]. This approach consists in automatically adapting the game in real-time to maintain patient's motivation on the one hand. On the other hand, this makes it possible for the therapist to supervise different patients and remotely when they return home.

For instance, Rabin et al. [8] propose a technique for difficulty adaptation based on an assessment exercise of the player's function that is carried out at the start of each game session. The inputs for adaptation are: the reached zone, wrist weight, grasp pressure and session date. The difficulty can be increased during the game session by making the target area progressively smaller and by adjusting the speed and range of targets. Although the evaluation of the technique included the player's motivation, the latter has not been involved in the decision on adaptation.

Pirovano et al. [10] focus on an intelligent game engine used to adapt games that are based on Wii Balance Board and Kinect. For instance, in Fruit Catcher game the player must catch fruits falling from the top of a tree using the basket. The difficulty is adapted by adjusting the number of falling fruits and baskets, fruit size, fruit weight as well as the falling frequency. The online adaptation 
is founded on a quest bayesian model that depends on the desired patient's performance (hit ratio in the game). This model was built using empirical data of healthy players' performance. However, as stroke patients performance can highly vary depending on their daily health conditions, this model should also be evaluated by including stroke patients' performance.
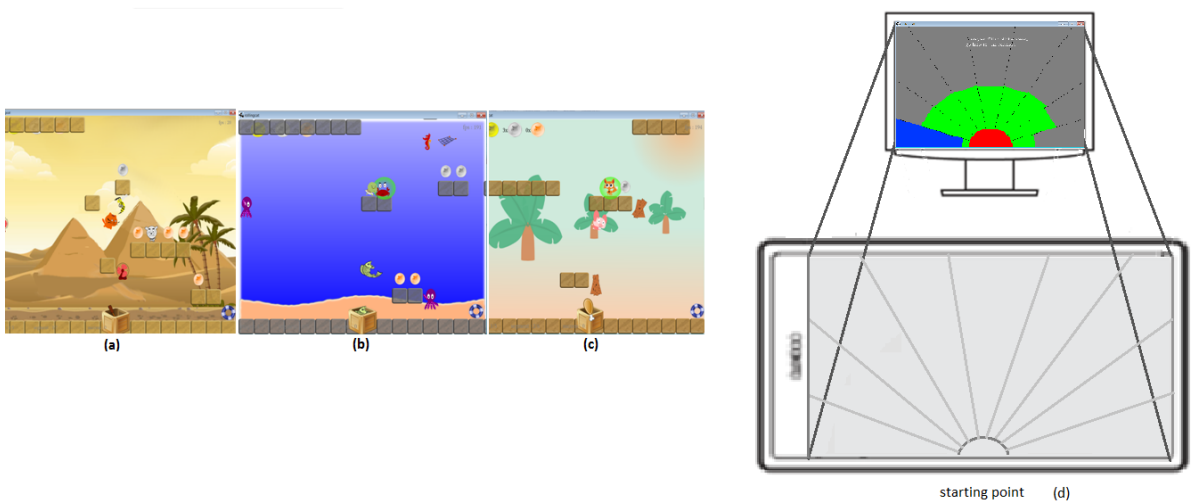

Fig. 1. Assessment procedure: patient is asked to cover up all the workspace by going to 9 different directions (d); PRehab game versions: Cat game (a), Turtle game (b) and Rabbit game (c)

In these studies, the adaptation inputs are mainly (i) the player's performance such as his/her final score [10], movement accuracy, range of motion and latency [9], reached zone, wrist weight and grasp pressure [8] as well as (ii) biomechanical measurements such as mean frequency of the position and force of signal [11]. Indeed, most difficulty adaptation techniques in upper-limb rehabilitation games are inspired by motor learning theories. These techniques only focus on the maximization of the effort during the rehabilitation session. However, the patient's motivation and engagement are among the key factors for recovery. In existing rehabilitation games, the patient's fatigue and motivation have been considered only to evaluate the game successfulness, but without including them in the decision on game adaptation [11]. Indeed, most studies attempted to show the utility of the game in increasing the training outcomes (e.g. motor control and shoulder/grasp strength) [8] as well as its usability and acceptability [10]. Only few works, namely Cameirao et al. [9], evaluated the effect of difficulty adaptation on patients' training outcomes but without considering their fatigue and motivation.

Our contribution consists in a generic adaptation technique for rehabilitation games. It depends on the generation of adaptive "pointing tasks" which represent the main player's activity in the game. The generation of tasks is based on the player's profile and performance. Unlike the previous works, the proposed tech- 
nique focuses on the maximization of the effort while taking into consideration the patients' conditions, in particular their abilities, fatigue and motivation.

\section{Player's Profile}

To automatically adapt the difficulty in a rehabilitation game, it is important to have a computational model of patients' motor abilities. The difficulty adaptation module requires the assessment of the current player's abilities to compute this model called the "ability zone" [12].

The ability zone represents the area where the patient can effectively carry out movements on a 2D workspace (e.g. graphics tablet). In general, this workspace has a dimension of 1-1.5 m. In fact, patients can be unable to reach very far targets from the starting point position or targets in such side of the workspace.

The ability zone of a patient in a workspace is modelled using a matrix of a dimension $m \times n$, where $m$ represents the number of rows and $n$ the number of columns. Each cell value represents an easiness score for the patient to reach the mapped area of the workspace. In [13] we proposed a bio inspired method to compute the ability zone matrix. Following this model, cells of the matrix contain digital pheromones that indicate whether the corresponding areas of the workspace were reached by the patient and with which quality of movement.

The ability zone is constructed during the assessment step before the play and can be updated during it. During the assessment exercise, the patient is asked to cover up all the workspace by going to 9 different directions as shown in Fig. 1 (d). All reached areas of the workspace are considered attainable and the others difficult. This data is used to compute the ability zone that helps the adaptation process to determine both challenge and assistance areas for the patient. Indeed, we consider all targets placed at areas inside the ability zone easy and the others difficult to reach.

\section{Difficulty Adaptation Technique}

The rehabilitation game is structured as a set of levels. Each game level which corresponds to a therapeutic session ( 20 to $45 \mathrm{~min}$ ) is defined by an objective that the player may achieve to progress in the game scenario. The level is composed of a set of game scenes. The latter is what the player's perceive during the interaction. Each game scene of the level contain a sequence of targets that the player's may reach to progress in the level. According to the position and range of the target, the patient may find it difficult or easy to reach.

The difficulty adaptation is based on the offline generation of pointing tasks that depends on the player's profile. During the game session, the game level is dynamically adapted according to the patient's performance and motivation. Next, we first describe the offline generation of pointing tasks and then the online adaptation of the game level. 


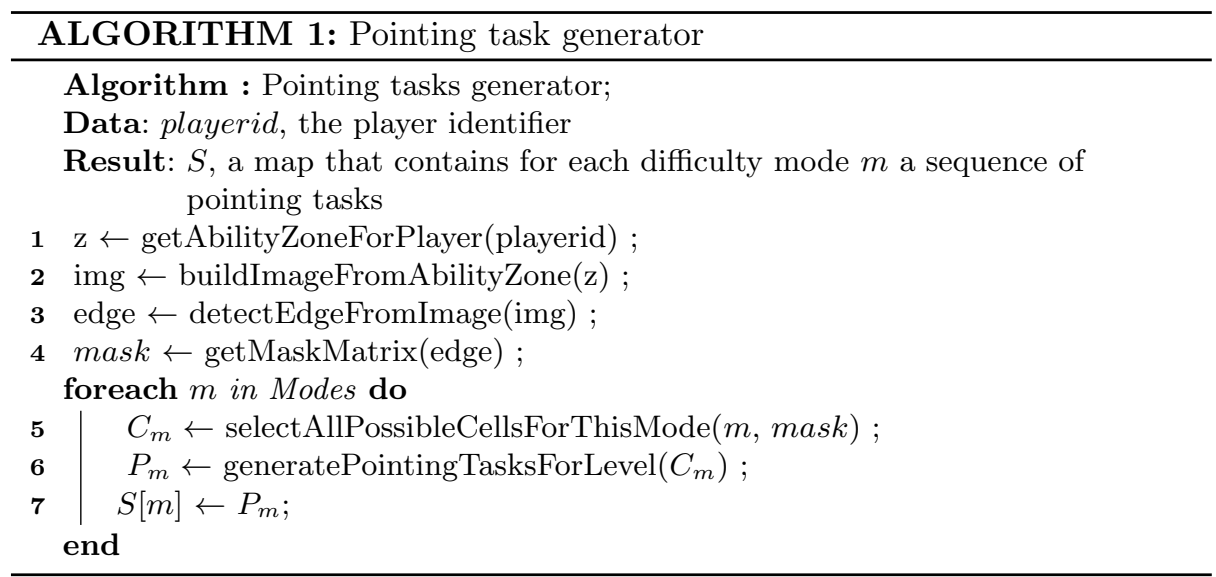

\subsection{Pointing Tasks Generation}

The pointing tasks generator is presented in Algorithm 1. This algorithm takes as an input the player's identifier playerid to retrieve the current ability zone (line 1). The obtained image of the ability zone is then used to determine difficult and easy areas of the workspace for the patient (lines 2 to 4 ). In fact, the edge of the ability zone image is used to compute the mask matrix that helps to determine the difficulty of pointing tasks.

For each predefined difficulty mode, a list of all potential areas is computed (line 5). This list is filtered using a heuristic search defined in Algorithm 2. The heuristic search is based on a Monte Carlo tree search to select the "best" pointing tasks for a game level (line 6). Next, we describe in details the main steps of the algorithm.

Step 1: Generating pointing tasks per difficulty mode The difficulty adaptation module uses the ability zone of the patient in order to determine the difficulty of pointing tasks. Since the ability zone is a matrix where each cell contains the digital pheromone intensity, we build an image representing this matrix where digital pheromone's intensity is translated to a grey scale color. Fig. 2 (2) is a simple example of the obtained image from the ability zone of Fig. 2 (1). The edge of this image represents the farthest possible area that the patient has reached. In fact, we consider the current abilities of the patient in order to challenge him/her. We assume that this challenge will have a sustainable training effect that can consequently increase the patient's range of motion.

To detect the edge in the ability zone image, a Sobel operator is used [14]. Sobel is an algorithm for edge detection in image processing used for images with high frequency variations. It emphasizes regions of high spatial frequency that correspond to edges. The operator is based on a pair of $3 \times 3$ convolution kernels $k_{x}=(-1,0,1 ;-2,0,2 ;-1,0,1)$ and $k_{y}=(1,2,1 ; 0,0,0 ;-1,-2,-1)$. These kernels are used to compute the gradient of each pixel of the image vertically $\left(G_{x}\right)$ 
and horizontally $\left(G_{y}\right)$. These measurements are used to compute the gradient magnitude on the basis of the following formula : $|G|=\sqrt{\left(G_{x}\right)^{2}+\left(G_{y}\right)^{2}}$.

Using the obtained gradient, we compute the mask matrix $(A)$ that helps the adaptation module to determine challenge and assistance areas as follows:

$$
A(i, j)=\left\{\begin{array}{l}
\text { true if }(G(i, j)>t) \\
\text { false otherwise }
\end{array}\right.
$$

Where $t$ is a threshold parameter to determine to what extent the movement frequency is considered. For example, given a gradient threshold $t=0.4$ the obtained edge of the ability zone image of Fig. 2 (1) is shown in Fig. 2 (3).

The mask matrix is used to determine possible targets in different difficulty modes. These modes are interpreted as follow:

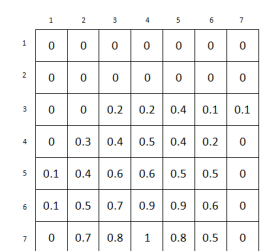

(1)

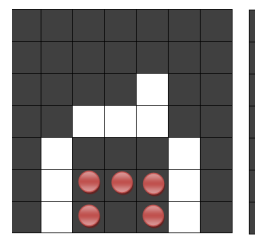

Easy

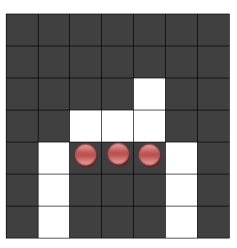

Medium

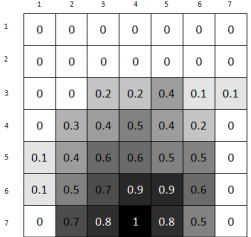

(2)

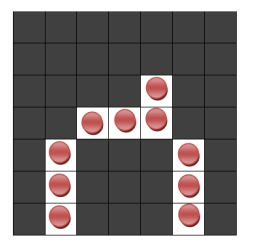

Difficult

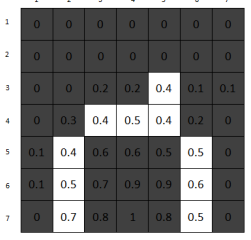

(3)

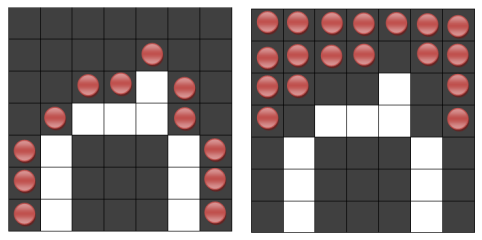

Very Difficult

Expert

Fig. 2. Example of ability zone (1), obtained image (2) and its edge (3); possible targets in the scene according to the difficulty mode (4)

- Easy: in this case the target is placed very close to the starting point.

- Medium: the target in this case is placed at the nearest bottom area from the edge of the image. The objective is to decrease the difficulty in order that the patient will be able to reach targets.

- Difficult: a target is considered as difficult when placed at the farthest reached area by the patient. It is the edge of the ability zone image determined by Sobel operator.

- Very difficult: a target is considered as very difficult when placed in the outside of the ability zone.

- Expert: the target in this mode is very far from the edge of the ability zone image. 
Step 2: Selecting pointing tasks for a game level The above presented process produces a mapping that links each difficulty mode to the areas of the workspace where pointing tasks with equivalent difficulty can be generated. The set of possibilities is too large and only few pointing tasks should be selected for the level. In [13] we focused on a random selection of pointing tasks. This often results in poor game scenes when for instance rules of symmetry and distribution are not taken into account. Moreover, from training perspective, using random selection for an easy level can produce targets that are very easy to reach. However, it is important in rehabilitation to assist the patient by decreasing the difficulty to the degree that he/she will be able to reach the target. This can help the patient to accomplish tasks with more important amplitude, which may consequently increase their range of motion.

To answer this issue, in this work the decision on the selection of targets is founded on heuristic search. In particular, a Monte Carlo Tree Search (MCTS) algorithm is used to generate the most suitable sequence of pointing tasks that takes into account both training and gaming constraints. The objective is to make a tradeoff between the enhancement of the training outcomes and the playability of the level to maintain the patient's motivation.

MCTS is an anytime search algorithm used to find optimal decisions by building a search tree from random samples in the decision space [15]. The general algorithm of MCTS is based on two policies: (i) tree policy to select or create the best leaf node within the search tree and (ii) default policy that produces a value estimate following the simulation of the action.

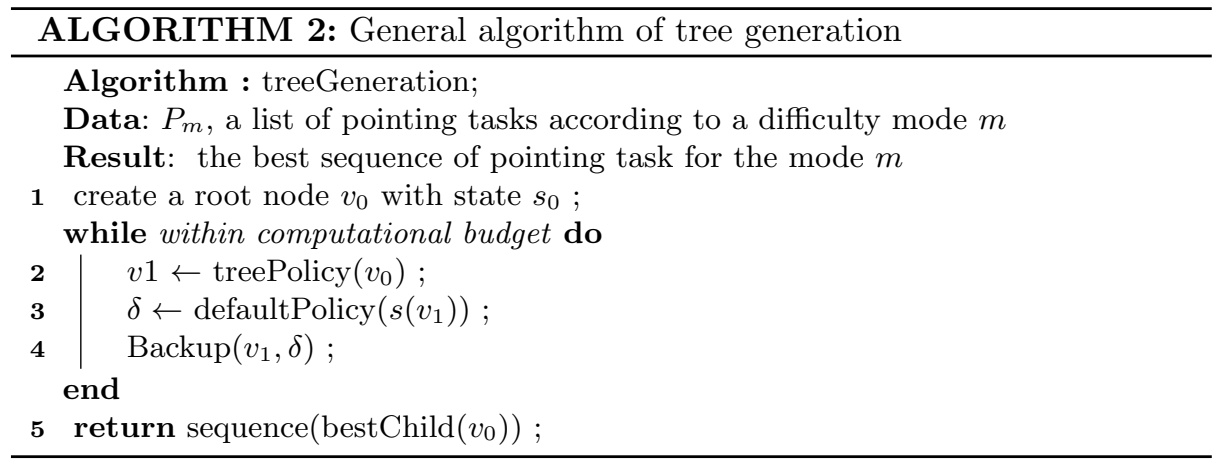

The pointing task generator builds five search trees according to difficulty modes. Each node of the tree represents a state of the game level which is a pointing task. The node contains information about the rewards as well as the number of visits of this node. The search tree involves iteratively building a tree until a budget is reached. The latter represents: (i) the given time to find a solution (number of iterations) and (ii) the volume (i.e number of tasks) of the level. 
The pseudo code of the tree generation is given in Algorithm 2. This algorithm takes as an input a list of pointing tasks according to a difficulty mode. The first step of the tree search consists in selecting a root node $v_{0}$ which represent the initial target at state $s_{0}$ (line 1 ). Then, within the computational budget the algorithm iteratively build the tree. The tree policy aims to select the best leaf node (line 2). The default strategy is run to simulate the added node (line 3 to 4 ), where $\delta$ is the reward for the last reached state. The result of the overall search (line 5) is sequence( bestChild $\left(v_{0}\right)$ ) which represents the obtained sequence of nodes that leads to the best leaf node from the root node $v_{0}$. In fact, the result consists in the sequence of all selected best nodes during the search.

The tree search produces a sequence of pointing tasks selected to maximize the reward function. This consists in selecting the best node (i.e. a target) on the basis of Upper Confidence Bounds for Trees (UCT) method. The best node is selected according to its UCT value given by the following formula [16]:

$$
V=\overline{R_{j}}+\sqrt{\frac{2 \cdot \log (n+1)}{n_{j}+\epsilon}}
$$

Where $\overline{R_{j}}$ is the average rewards from node $j$ that are understood to be within $[0 ; 1], n$ is the number of the times the current parent node has been visited, $n_{j}$ is the total number of times that the child node $j$ has been visited and $\epsilon$ is a tunable bias parameter.

Indeed, in this formula the reward term $\bar{R}_{j}$ encourages the exploitation of nodes with higher-rewards and the right hand term $\sqrt{\frac{2 \cdot \log (n+1)}{n_{j}+\epsilon}}$ encourages the exploration of less visited choices. UCT insures the property of equilibrium between exploitation and exploration. In fact, it is important to suggest to the patient tasks that increase the reward function while checking his/her capability to explore the workspace. This can consequently evolve the patient's ability zone.

The reward value $R$ for a node $j$ is given by the following formula:

$$
R=\frac{1}{2}(\alpha . \operatorname{distance}(j)+\beta . \operatorname{shift}(j))
$$

It depends on: (i) the importance of reaching a target in such position of the workspace given by the function $\operatorname{distance}(j)$; and (ii) $\operatorname{shift}(j)$ function that determines to what extent the child node $j$ is left aligned in the game scene; where $\alpha+\beta=1$.

\subsection{Online Difficulty Adaptation}

The obtained sequences of pointing tasks are used to generate the game level. In fact, for each difficulty mode a sub-level is created. The next step consists in the online adaptation of the difficulty by suggesting to the patient game scenes 
of one of the generated sub-levels. This depends on the selected difficulty mode according to the patient's motivation during the game level.

We focus on a motivation model we introduced in [12]. This model depends on a window of success and failure to make decision on adaptation. The motivation model is inspired by the activation theory [17]. In this theory, stimulation is necessary and the use of an activation level is required in order to make an individual sufficiently motivated to perform tasks. As a measure of the level of activation, we consider the number of successive task success and failure during the level [12]. According to the patient's successes and failures, the motivation model makes the following decisions: (i) decrease the difficulty to avoid putting the player in repeated situations of failure during the game level; (ii) increase the difficulty when the player is in a situation of consecutive successes and finds the game very easy; and otherwise (iii) maintain momentarily the difficulty.

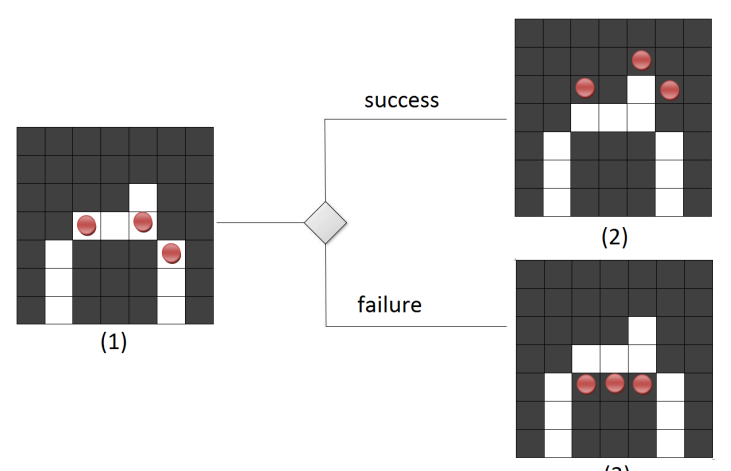

(3)

Fig. 3. Example of proposed scenes to a player

\subsection{Example}

The obtained image of the ability zone of Fig. 2 is used to compute the mask matrix. According to the difficulty mode, the possible targets positions are shown in Fig. 2 (4). Then, for each difficulty mode, a search tree is built in order to find the best sequence of pointing tasks for this difficulty mode. Fig. 3 shows an example of game scenes proposed to the player during the game session. First, the default difficulty mode is difficult (1). The window of success and failure $w=1$. Thus, if the player succeeds the objective of this scene, the next proposed scene is the very difficult one (2) otherwise come across the medium mode (3).

The adaptation technique has been implemented in a rehabilitation game we developed named PRehab. This game can be played using a computer mouse or a graphics tablet. Different versions of this game have been implemented by varying the aesthetics (ambiances): sea ambiance (Turtle game, see Fig. 1 (b)); 
desert ambiance (Cat game, see Fig. 1 (a)); and forest ambiance (Rabbit game, see Fig. 1 (c)). In these PRehab versions, the player is asked to eliminate the enemies that constitute the obstacles for the given main character (turtle, cat or rabbit). Besides, the player can collect different coins to increase his/her score points. To free the path, the player has to use objects from his/her virtual box to eliminate obstacles. From motor training perspective, the objective is to reach targets (enemies) that appear at different areas of the game scene.

At the start of the game level, the main character is placed at the first scene of the sub-level corresponding to the difficult mode. The game difficulty is then adapted according to the player's performance and motivation during the play. The adaptation in this case focuses on camera move in order to provide the player with adapted game scenes during the game level.

\section{Experiment}

The objective of the experiment is to study the impact of the proposed adaptation technique on patient's motivation and training outcomes. The experiment was conducted in the hospital of Grau du Roi of Montpellier, France.

We focused on a repeated-measures single-blinded design in which each patient is asked to perform an assessment exercise and then play a game version that is randomly selected. Patients play the game versions in different sequences. Only the difficulty strategy that was studied. Three conditions of this independent variable were considered:

- Dynamic difficulty adaptation (DDA): it is the proposed approach based on online difficulty adaptation. The difficulty strategy consists in increasing and/or deceasing the difficulty during the play according to the patient's profile and motivation in Turtle game.

- Incremental difficulty adaptation (IDA): this strategy consists in progressively providing the patient with the easiest to most difficult tasks in Rabbit game. It does not consider the patient's profile and motivation.

- Without a difficulty adaptation strategy (Random) in Cat game.

The objective is to compare the effects of the tree conditions (DDA, IDA and Random) on the player's training outcomes and motivation. The null hypotheses have be defined as follow: HO. the difficulty strategy has no effect on measures outcomes. The main measures outcomes were: the number of tasks, number of successful tasks, distance, velocity, perceived game duration and perceived difficulty. Moreover, interviews with therapists and patients have been recorded at the end of the game sessions. In particular, we asked both patients and therapists whether the game is adapted to patients' abilities, whether the objectives are clear and the acceptance of using the games in their daily rehabilitation sessions.

All participants used the same device: a graphics tablet Intus A3. The three versions of PRehab have different aesthetics in order to make it easy to the patient to differentiate the three game versions. The three games are based on the same mechanics and the main activity of the player is to reach targets. In 
fact, in our previous clinical tests we observed that the patient has difficulties to make distinctions between the different software that are based on the same interface.

Inclusion criteria of subjects were not restrictive. The only criterion was the ability to use the game device (mouse of graphics tablet) and play the game (without strong cognitive deficits). Seven post stroke patients aged 38 to 73 years old $(61.57 \pm 12.06)$ were provided with the three game versions and were asked to complete tasks using the impaired arm. Only a patient who played the games in bi-manual due to strong difficulties. This group of patients who have limited capabilities due to stroke, was composed of 5 men and 2 women. The number of weeks after stroke was $(15.71 \pm 7.47)$.

The experiment was conducted during two weeks in which each participant was asked to perform an assessment exercise and then play a game version during a rehabilitation session $(20 \mathrm{~min})$. Each patient carried out three rehabilitation sessions, one each day at approximately the same hour. Each game version generated levels that allow carrying out a bounded number of pointing tasks. After each game session, a questionnaire was proposed to patients to report their experience.

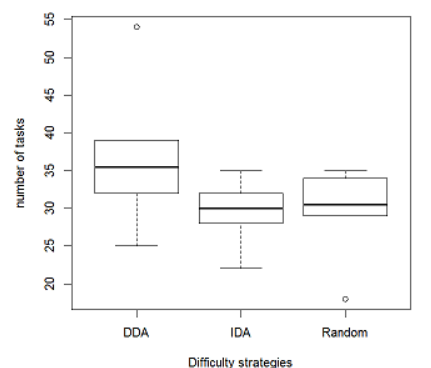

(a) Number of tasks

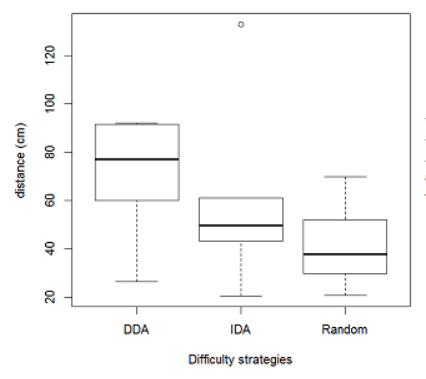

(c) Total distance

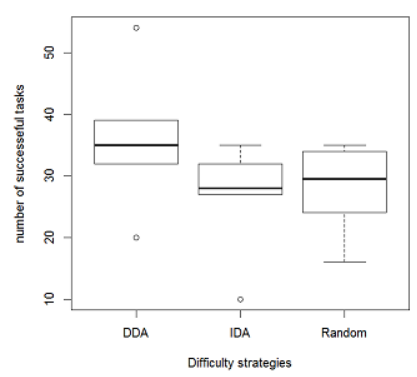

(b) Number of successful tasks

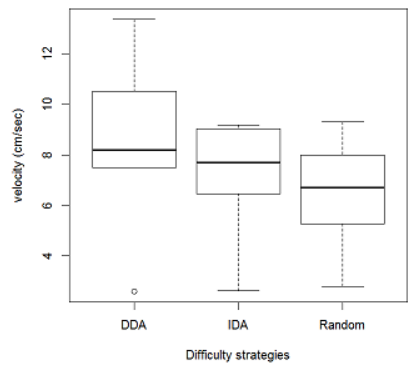

(d) Velocity

Fig. 4. Measures outcomes for the three difficulty strategies 


\section{Results and Discussion}

All statistical analysis were performed using R (http://www.r-project.org) version 2.12.0. To analyze the effect of the three difficulty strategies on measures outcomes, a one-way repeated measures ANOVA was used. When an analysis revealed significant differences, post-hoc pairwise comparisons based on Tukey HSD test were used to locate the specific differences, where $p<0.05$ was accepted as significant. Before the analysis of mean and variance, a Kolmogorov-Smirnov normality test was initially used. In addition, Friedman test was used to reject hypotheses for ordinal measures.

A repeated-measures analysis of variance indicated significant mean differences between the three difficulty strategies for three dependant variables: the number of tasks $\left(D F_{d}=10, F=5.85, p<0.02, \eta^{2}=0.53\right)$; the number of successful tasks $\left(D F_{d}=10, F=7.70, p<0.009, \eta^{2}=0.60\right)$; and the total distance $\left(D F_{d}=10, F=4.39, p<0.04, \eta^{2}=0.46\right)$.

The number of tasks carried out by the patients was significantly more important in the Turtle game (with DDA strategy) compared with the two other games (with IDA and Random strategies). The HSD test indicates a significant difference in number of tasks between DDA and IDA $p<0,047$ and between DDA and Random $p<0,058$ and no significant difference has been recorded between Random and IDA (see Fig. 4 (a)). In fact, as DDA is based on the control of patient's motivation through performances, this can increase patients' engagement and prevent their tiredness. Therefore, the patients put more efforts, increasing thus the number of tasks.

The mean differences in the total distance was significant. It was significantly more important in DDA compared with the other strategies, especially the Random strategy with $p<0,007$ (see Fig. 4 (c)). Therefore, DDA has increased the amplitude of movement which is an important factor in stroke patients' recovery.

Despite the game based on DDA was challenging, the number of successful tasks was more important in this game compared with the other game versions. The difference in the mean of number of successful tasks in the three game versions was statically significant (see Fig. 4 (b)). The differences between DDA and IDA $p<0,014$ as well as between DDA and Random $p<0,044$ were significant and no difference has been reported between Random and IDA.

Although the existing differences between the three strategies in terms of velocity (see the graph of Fig. $4(\mathrm{~d}))$, the ANOVA test was not significant $\left(D F_{d}=\right.$ $\left.10, F=4.03, p<0.051, \eta^{2}=0.53\right)$. Moreover, all players have reported their enjoyment when using games in rehabilitation. The perceived session duration for all games was different from the effective one $(20 \mathrm{~min})$. Indeed, most patients have reported that the session duration was about 3 to $15 \mathrm{~min}$. Although the existing difference between the strategies, the ANOVA test has not rejected the hypothesis for perceived duration $\left(D F_{d}=10, F=3.54, p<0.06, \eta^{2}=0.41\right)$.

Using a scale of five choices (0: not at all, 1: slightly, 2: moderately, 3: fairly and 4: extremely) the patients have reported their perceived difficulty of game tasks. The Friedman test has not rejected the hypothesis for the perceived difficulty as the number of subjects is less than 12 (Friedman chi-squared 
$=2.8 ; d f=2 ; p<0.24)$. Nevertheless, the median values show that the game based DDA was moderately difficult (2) as for the other games the difficulty was reported as fairly difficult (3) in IDA and slightly difficult (1) in Random strategy. Hence, we assume that although the game based on DDA was challenging, the patients have perceived that the game was not very difficult. This assumption should be confirmed in future experiments.

Furthermore, interviews with patients have been carried out at the end of the experiment and observations on patients verbal reactions were recorded. All patients have reported their motivation and acceptance of using games in rehabilitation. In particular, some patients who have pains, stressed to continue to play the game as they were motivated. Finally, the patients preferred the game version based on DDA as this game was ranked at the first and second position more times than the other two games.

\section{Conclusion}

Our contribution focuses on a dynamic difficulty adaptation (DDA) technique that aims to increase the patients' training outcomes while maintaining their motivation. The study focused on the evaluation of the effects of proposed strategy on training outcomes and motivation compared with control strategies. The results of the experiment show that DDA has increased the number of tasks, number of successful tasks and total distance in levels compared with the control strategies. This is an important factor allowing to increase the training volume in stroke patients. This study showed the importance of software control and adaptation in the case of serious games, especially for motor rehabilitation. In this area, the adaptation plays an important role to make a tradeoff between the utility of the game and its ability to create an enjoyable game experience.

Finally, some limits of the study have been studied. In particular, the number of subjects we were able to include in the study was limited. Moreover, in order to help patients to make the difference between the three difficulty strategies, we focused on a game with three ambiances. This bias has been considered not important in the study. In fact, the patients have reported the similarity between the three games and we therefore assumed that the bias has not influenced the study outcomes. In our future work, we will increase the number of subjects who should have different profiles. In addition, a particular focus will be in enhancing the difficulty strategy by manipulating time constraints on movements. This can be important for patients who have an advanced recovery stage.

\section{References}

1. Kwakkel, G., Wagenaar, R., Koelman, T., Lankhorst, G. J., Koetsier, J. C.: Effects of intensity of rehabilitation after stroke a research synthesis. Journal of Stroke, vol. 28, pp. 1550-1556, Am Heart Assoc (1997)

2. Cirstea, M., Levin, M.: Improvement of arm movement patterns and endpoint control depends on type of feedback during practice in stroke survivors. Journal of Neurorehabilitation and Neural Repair, vol. 21, pp. 398-411, Sage Publications (2007) 
3. Dobkin, B.: Rehabilitation after stroke. New England Journal of Medicine, vol. 352, pp. 1677-1684, Mass Medical Soc (2005)

4. Howcroft, J., Klejman, S., Fehlings, D., Wright, V., Zabjek, K., Andrysek, J., Biddiss, E.: Active video game play in children with cerebral palsy: Potential for physical activity promotion and rehabilitation therapies. Archives of physical medicine and rehabilitation, vol. 93, pp. 1448-1456, Elsevier (2012)

5. Malone, T. W. : What makes computer games fun?, vol. 6, pp. 258-277 (1980) T. W. Malone, What makes computer games fun?

6. Csikszentmihalyi, M. : Beyond Boredom and Anxiety: Experiencing Flow in Work and Play, San Francisco: Jossey-Bass (2000)

7. Avila-Sansores, S., Orihuela-Espina, F., Enrique-Sucar, L.: Patient Tailored Virtual Rehabilitation. In: Converging Clinical and Engineering Research on Neurorehabilitation, pp. 879-883. Springer (2013)

8. Rabin, B., Burdea, G., Hundal, J., Roll, D., Damiani, F.: Integrative motor, emotive and cognitive therapy for elderly patients chronic post-stroke A feasibility study of the BrightArm rehabilitation system. In: 2011 International Conference on Virtual Rehabilitation (ICVR), IEEE (2011)

9. Cameirão, M.S., Badia, S.B., Oller, E.D., Verschure, P.: Neurorehabilitation using the virtual reality based Rehabilitation Gaming System: methodology, design, psychometrics, usability and validation. Journal of neuroengineering and rehabilitation, vol. 7, pp. 48, BioMed Central Ltd (2010)

10. Pirovano, M., Mainetti, R., Baud-Bovy, G., Lanzi, P., Borghese, N.: Self-adaptive games for rehabilitation at home. In: 2012 IEEE Conference on Computational Intelligence and Games (CIG), pp. 179-186 IEEE (2012)

11. Mihelj, M., Novak, D., Milavec, M., Ziherl, J., Olenšek, A., Munih, M.: Virtual rehabilitation environment using principles of intrinsic motivation and game design. Journal of Presence: Teleoperators and Virtual Environments, vol. 21, pp. 1-15, MIT Press (2012)

12. Hocine, N., Gouaich, A., Di Loreto, I., Joab, M.: Motivation based difficulty adaptation for therapeutic games. In: 2011 IEEE 1st International Conference on Serious Games and Applications for Health (SeGAH), pp. 1-8, IEEE (2011)

13. Gouaich, A., Hocine, N., Van Dokkum, L., Mottet, D.: Digital-pheromone based difficulty adaptation in post-stroke therapeutic games. In Proceedings of the 2nd ACM SIGHIT International Health Informatics Symposium, pp. 5-12, ACM (2012)

14. Vincent, O.R., Folorunso, O.: A descriptive algorithm for sobel image edge detection. In Proceedings of Informing Science \& IT Education Conference (InSITE), pp. 97-107 (2009)

15. Tavener, S., Perez, D., Samothrakis, S., Colton, S.: A Survey of Monte Carlo Tree Search Methods. In IEEE Transactions on Computational Intelligence and AI in Games, vol. 4, IEEE (2012)

16. Kocsis, L., Szepesvári, C.: Bandit based monte-carlo planning. In Machine Learning: ECML 2006, pp. 282-293, Springer (2006)

17. Scott, W.: Activation theory and task design. In Organizational Behavior and Human Performance (1966)

18. Atkinson, J. W.: Motivational determinants of risk-taking behavior. Psychological review, vol. 64, American Psychological Association (1957) 